\title{
Testando mudanças estruturais na regra de Taylor: um estudo empírico para o Brasil (2000-2011)
}

\author{
Nadja Simone Menezes Nery de Oliveira ${ }^{1}$ \\ Edson Ramos de Medeiros ${ }^{2}$ \\ Gabriela Bezerra de Medeiros ${ }^{3}$ \\ Edilean Kleber da Silva Bejarano Aragón ${ }^{4}$ \\ Umberto Antonio Sesso Filho ${ }^{5}$
}

\begin{abstract}
Resumo: O presente estudo investiga a existência de mudança estrutural na regra de política monetária adotada pelo Banco Central do Brasil no período de 2000 a 2011, isto é, a pesquisa busca investigar alterações na dinâmica de definição da taxa Selic pelo Banco Central brasileiro no período de metas de inflação. Para este fim foi utilizada a metodologia proposta por Bai e Perron (2003) que, por sua vez, consiste em estimar a ocorrência de mais de um ponto de quebra estrutural em uma data desconhecida. Os resultados obtidos mostram que desde adoção do regime de metas inflacionárias os coeficientes da regra de política monetária adotada pelo BACEN durante o período de 2000-2011não permaneceram constantes. O teste de mudanças estruturais indicou que há evidências de duas quebras estruturais nos coeficientes da função de reação do Banco central do Brasil no período de metas para a inflação, as datas das mudanças estruturais obtidas foram 2004.2 e 2007.10.
\end{abstract}

Palavras-chave: Regra de Taylor; Função de Reação; Mudanças Estruturais; Metas de Inflação.

JEL: F42; E43; E58.

1 Mestranda em Economia Regional pelo Programa de Pós-Graduação em Economia da Universidade Estadual de Londrina (PPE/UEL). E-mail: nadja_menezes@hotmail.com

2 Mestre em Economia Regional pelo Programa de Pós-Graduação em Economia da Universidade Estadual de Londrina (PPE/UEL).E-mail: edsonrmedeiros@yahoo.com.br

3 Doutoranda em Economia Aplicada pelo Programa de Pós-Graduação em Economia (PPGE/UFRGS) da Universidade Federal do Rio Grande do SulE-mail: gabriela.bm@hotmail.com

4 Pós-Doutor (PhD) em Economia Aplicada pelo Programa de Pós-Graduação em Economia (PPGE/UFRGS) da Universidade Federal do Rio Grande do Sul. Professor do Programa de Pós-Graduação em Economia (PPGE/UFPB) da Universidade Federal da Paraíba. E-mail: edilean@hotmail.com

5 Doutor em Economia Aplicada pelo Programa de Pós-Graduação em Economia da Escola Superior de Agricultura Luiz de Queiroz (ESALQ/USP). Professor do programa de Pós-Graduação em Economia da Universidade Estadual de Londrina (PPE/UEL). E-mail: umasesso@uel.br 


\title{
Testing structural changes in the Taylor rule: an empirical study for Brazil (2000-2011)
}

\begin{abstract}
This study investigates the existence of structural change in the monetary policy rule adopted by the Central Bank of Brazil from 2000 to 2011, ie, the research seeks to investigate changes in the dynamic setting of the Selic rate by the Central Bank of Brazil in the period inflation targeting. For this purpose was the methodology proposed by Bai and Perron (2003) that, in turn, is to estimate the occurrence of more than one structural break point at a date unknown. The results show that since the adoption of inflation targeting regime the coefficients of the monetary policy rule adopted by the Central Bank during the 200o-2011não remained constant. The test of structural changes indicated that there is evidence of two structural breaks in the coefficients of the reaction function of the central bank of Brazil from inflation targets, dates of structural changes obtained were 2004.2 and 2007.10.
\end{abstract}

Key-words: CTaylor Rule; Reaction Function, Structural Changes, Inflation Targeting.

JEL: F42; E43; E58

\section{Introdução}

O presente estudo tem por objetivo investigar a existência de mudança estrutural na regra de política monetária adotada pelo Banco Central do Brasil no período de 2000 a 2011, isto é, a pesquisa buscará investigar alterações na dinâmica de definição da taxa Selic pelo Banco Central brasileiro no período de metas de inflação. Para tal, será utilizada a metodologia proposta por Bai e Perron (2003) que, por sua vez, consiste em estimar a ocorrência de mais de um ponto de quebra estrutural em uma data desconhecida.

A regra de política monetária proposta inicialmente por Taylor (1993) estabelece que a taxa de juros definida pelo Banco Central depende fundamentalmente da taxa de inflação e do hiato do produto. Desde então, vários estudos passaram a ser realizados usando este mesmo princípio, nos quais se busca estimar uma função de reação capaz de descrever a evolução do instrumento de política monetária do Banco Central, a taxa básica de juros, ao longo do tempo.

Clarida et al. (2000) propuseram uma importante modificação na regra de Taylor ao formularem uma regra de política monetária capaz de relacionar os ajustes da autoridade monetária na taxa de juros corrente com base nos valores futuros esperados da inflação e no hiato do produto. 
Na literatura nacional, alguns autores buscaram descrever uma regra de Taylor com o intuito de interpretar como se dá a condução da política monetária sob o regime de metas inflacionárias (ver, por exemplo, Minella et al., 2003; Barcellos Neto, 2003; Holland, 2005; Salgado et al., 2005; Soares e Barbosa, 2006).

Seis meses após os ataques especulativos investidos contra o Real em janeiro de 1999, que fizeram com que a taxa de câmbio nacional passasse de administrada para flutuante, o Brasil adotou o regime de metas de inflação. Com a implementação deste regime de política, o Banco Central do Brasil passou a alterar o instrumento de política monetária, a taxa Selic, com o principal objetivo de fazer com que a inflação convergisse à meta estabelecida pelo Conselho Monetário Nacional (CMN).

Um tema bastante importante que também vem sendo estudado na literatura econômica, porém pouco explorado na literatura nacional, é o exame na mudança dos parâmetros da função de reação do Banco Central. Alguns autores brasileiros abordam essa temática, por exemplo, o trabalho proposto por Lima et al. (2007), no qual através de um modelo markoviano de mudanças, estima as funções de reação para o período pós-plano Real e localizaram existência de dois regimes para o período de agosto de 1999 a janeiro de 2006. Barcellos Neto e Portugal (2007) encontraram evidências empíricas de que na administração do Banco Central do Brasil por Henrique Meirelles, a taxa básica de juros (Selic) tem reagido menos aos desvios na inflação esperada em relação a sua meta e mais a variações na taxa de câmbio quando comparada a administração de Armínio Fraga.

Portanto, a motivação do presente trabalho se deve, principalmente pela falta de consenso de um modelo mais apropriado para caracterizar o comportamento da política monetária do Brasil a partir da implantação do sistema de metas de inflação.

Neste sentido, esta investigação tem por objetivo estimar uma regra de Taylor para o regime de metas brasileiro e, por meio de uma metodologia para seleção de quebras estruturais, identificar as possíveis mudanças de regimes na dinâmica de definição da taxa básica de juros da economia, a taxa Selic, por parte da autoridade monetária no período de 2000 a 2011. Para isso, utilizamos a metodologia proposta por Bai e Perron (2003), através do teste de múltiplas quebras estruturais para datas desconhecidas.

\section{Revisão da Literatura}

Em seu artigo, John B. Taylor (1993) apresentou uma equação linear simples entre o comportamento das taxas internas de juros de curto prazo dos Estados Unidos, o desvio da inflação em relação a uma meta de inflação estabelecida 
e o desvio do produto real em relação ao produto potencial para o período de 1987 a 1992. Taylor procurou mostrar que a política monetária deve ser conduzida por meio de regras transparentes e críveis, uma vez que acreditava que esta era a forma mais eficaz de atingir os melhores resultados conjuntos de desempenho econômico. De acordo com Taylor (1993), o desempenho da taxa de juros nos EUA poderia ser representado por uma relação linear simples com a taxa de inflação $\left(\pi^{*}\right)$, uma taxa de juros de equilíbrio $\left(r^{*}\right)$ e uma soma ponderada entre dois desvios: a diferença entre a taxa de inflação (medida pelo deflator do PIB) e a meta de inflação e o desvio percentual do PIB do seu potencial. Essa relação linear pode ser expressa como:

$$
i_{t}=\pi_{t}+r^{*}+0,5\left(\pi_{t}-\pi^{*}\right)+0,5\left(y_{t}\right)
$$

em que $i_{t}$ é a taxa básica de juros, $r^{*}$ é a taxa real de juros de equilíbrio, $\pi$ é a taxa de inflação (medida pelo deflator do PIB), $\pi^{*}$ é a meta de inflação e $y$ é o hiato do produto (isto é, o desvio percentual do produto real em relação ao produto potencial).

A partir do trabalho de Taylor (1993), inúmeros estudos, tanto teóricos quanto empíricos, foram realizados para a obtenção de funções de reação. Dentre os quais, destaca-se o trabalho de Clarida, Galí e Gertler (1998) que estimaram funções de reação para os bancos centrais de países industrializados. Os autores dividiram esses países em dois grupos o $\mathrm{G} 3$ composto por Alemanha, Japão e EUA e o E3 formado por Itália, França e Reino Unido. Esse estudo evidenciou que os bancos centrais do grupo $\mathrm{G}_{3}$ têm perspectiva de futuro (forward-looking), eles respondem à inflação prevista como oposição à perspectiva backward-looking, adotada por Taylor (1993), onde os valores passados de inflação e produto foram utilizados no modelo.

A regra básica estimada para cada um dos países é dada por:

$$
r_{t}=(1-\rho) \alpha+(1-\rho) \beta \pi_{t+n}+(1+\rho) \gamma x_{t}+\rho+r_{t-1}+\varepsilon_{t}
$$

em que $r_{t}$ é a taxa de juros nominal, $\pi_{t+n}$ é a taxa de inflação no período $\mathrm{t}+\mathrm{n}$, $x_{t}$ é o hiato do produto e $\varepsilon_{t}$ é o termo de erro.

Através de uma versão mais sofisticada, estes autores chegaram à conclusão de que os países que formam o grupo G3 possuem metas implícitas de inflação. Levando em consideração os dados da amostra, os mesmos defendem explicitamente que um sistema de metas de inflação é possivelmente superior a um sistema de câmbio fixo, em termos de desempenho conjunto das taxas de inflação e de crescimento econômico.

Judd e Rudebusch (1998) analisaram uma função de reação à política monetária americana durante o período de 1970 até 1997, o objetivo era analisar o período correspondente a três presidentes distintos e a partir dessa estimação avaliar a hipótese se a troca de diferentes presidentes 
alterava a conduta do instrumental de política monetária. Para tal, os autores subdividiram a amostra em três partes: o período cuja gestão estava a cargo de Arthur Burns (1970. Q1-1978. Q1), Paul Volcker (1979. Q3-1987. Q2), e Alan Greenspan (1987. Q3-1997. Q1). Vale ressaltar que durante o período total de análise a presidência do Fed também esteve sob a gestão de Miller (1978. Q2-1979. Q2), que devido a sua curta duração foi desconsiderada pelos autores. Nesse trabalho foram localizados bons indícios de que os movimentos nas taxas de juros são consistentes com uma política monetária que almeja inflação baixa no longo prazo e crescimento econômico próximo de seu potencial de curto prazo.

No entanto, os resultados dessa análise diferiram da especificação original de Taylor (1993) em dois importantes aspectos. As taxas de juros parecem reagir mais intensamente aos desvios do PIB ao contrário do que o artigo original supunha, ao passo que a velocidade do ajuste parece ser mais suave do que supôs Taylor. Ademais, foram encontrados sinais de que a regra de Taylor se adapta melhor ao período em que as decisões do Fed estiveram sob o comando de Alan Greenspan.

\subsection{Resultados empíricos para o Brasil}

Em relação ao Brasil, existem vários trabalhos que procuram realizar estimações de funções de reação com a finalidade de captar o comportamento da autoridade monetária em sua condução de política monetária. Dentre esses podemos citar Minella et al. (2003) que estimaram uma função de reação para o Banco Central do Brasil que relaciona a taxa de juros de curto prazo a desvios da taxa de inflação esperada em relação à meta de inflação, permitindo alguma suavização da taxa de juros, e ainda, ao hiato do produto e aos movimentos da taxa de câmbio. A relação destas variáveis pode ser melhor visualizada com base na seguinte especificação:

$$
i_{t}=\rho i_{t-1}+(1+\rho)\left[\alpha_{0}+\alpha_{1} D_{j}+\alpha_{2} y_{t-1}+\alpha_{3} \Delta e_{t-1}\right]+\varepsilon_{t}
$$

onde $i_{t}$ é a taxa nominal de juros (Selic) estabelecida pelo Comitê de Política Monetária (COPOM), $D_{j}$ é a média ponderada entre os desvios no ano corrente e no ano seguinte da expectativa de inflação em relação à meta inflacionária, $y_{t-1}$ é o hiato do produto, $\Delta e_{t-1}$ é a variação da taxa de câmbio nominal e $\varepsilon_{t}$ é o termo de erro.

O hiato do produto é obtido pela diferença entre o produto atual e o potencial, sendo usado como proxy do produto atual a produção industrial mensal medida pelo IBGE, e o produto potencial estimado através do filtro HP, proposto por Hodrick e Prescott em estudo de 1997, que define uma tendência de longo prazo como uma média ponderada da série em análise. A amostra utilizada foi formada por dados mensais para o período de julho de 1999 a 
dezembro de 2002. Os autores utilizam ainda duas fontes de expectativa de inflação: a expectativa de inflação estimada pelo Banco Central do Brasil em seu Relatório trimestral de inflação e as expectativas obtidas a partir de um levantamento diário que o Banco Central realiza entre instituições financeiras e empresas de consultoria. Esse levantamento pergunta o que as firmas esperam da inflação de períodos determinados. Desse modo, Minella et al (2003) concluíram que, no período observado, o BACEN teve uma postura forward-looking, ou seja, reagiu agressivamente aos desvios das expectativas de inflação em relação à meta estabelecida.

Barcellos Neto (2003) estima uma função de reação do tipo Taylor (1993) para o Banco Central do Brasil no período sucedido pela adoção do regime de metas para a inflação, de acordo com a seguinte especificação:

$$
i_{c}=c+\beta_{1 t-j}^{i}+\beta_{2} D_{j}+\beta_{3} y_{t-1}+\beta_{4} I P C A l_{t-1}+\beta_{5} I P C A a_{t-1}+\varepsilon_{t}
$$

onde $i_{t}$ é a taxa Selic mensal (média do período), $D_{j}$ é o desvio ponderado da inflação esperada em relação à meta de inflação, $y_{t-1}$ é o hiato do produto defasado um período, $I P C A l_{t-1}$ é a soma móvel de 12 meses do Índice de Preços ao Consumidor Amplo (IPCA) para os preços livres e $I P C A a_{t-1}$ é a soma móvel de 12 meses do índice de preços ao consumidor amplo para os preços administrados.

Dentre os modelos desenvolvidos pelo autor esta equação sugere um excelente grau de explicação das variáveis às decisões do BACEN, pois se apresenta em sintonia com as diretrizes do regime de metas de inflação com câmbio flutuante, indicando que a autoridade monetária vem se comportando de modo consistente com o próprio sistema implementado no Brasil a partir do segundo semestre de 1999. Dessa forma, os resultados obtidos nesse estudo mostram que o BACEN leva em consideração em suas decisões de política monetária o comportamento dos desvios ponderados das expectativas de inflação em relação à meta do ano corrente e do ano seguinte, o comportamento do hiato do produto e o comportamento do IPCA diferenciado entre preços livres e administrados.

Holland (2005) estimou uma função de reação para o BACEN para o período de metas de inflação com o objetivo de examinar empiricamente se a política da autoridade monetária do Brasil sob um regime de câmbio flutuante tem respondido aos choques de câmbio através de sua função de reação. Em sua proposta a taxa de juros básica $\left(i_{t}\right)$, a taxa Selic, é uma função da expectativa de inflação $E\left(\pi_{t+n}\right)$, do hiato do produto $\left(x_{t}\right)$ e da taxa de câmbio real $\left(z_{t}\right)$, que pode ser observada na função seguinte:

$$
i_{t}=\phi\left\{\alpha+\beta E\left[\pi_{t+n} / \Omega_{t}\right]+\gamma E\left[x_{t} / \Omega_{t}\right]+\xi E\left[z_{t} / \Omega_{t}\right]\right\}+(1+\phi) i_{t-1}
$$

A amostra de dados é mensal e abrange o período de julho de 1999 até janeiro de 2005. Como metodologia este autor utiliza o método dos momentos 
generalizados (GMM). Os resultados obtidos por Holland (2005), foram os de que o Banco Central possui uma postura agressiva em relação ao controle inflacionário, visto que os valores obtidos dos coeficientes de inflação $(\beta)$ em sua estimação tiveram resultados bem superiores a 1 e estatisticamente significativos, e dado que os valores estimados para os coeficientes da depreciação cambial real $(\xi)$ não foram estatisticamente significativos, mostrando que a política monetária do Brasil não responde a depreciação na taxa de câmbio real.

Salgado et al. (2005) objetivando analisar os efeitos de mudanças de regime na determinação da taxa básica de juros para o período entre agosto de 1994 e dezembro de 2000 estimam uma função de reação para o BACEN fundamentada em um modelo TAR (Limiar Auto-Regressivo). Em particular, o modelo proposto pelos autores faz uso de um indicador de crises cambiais para analisar variações nas dinâmicas da taxa Selic em períodos com crises e fora de crises. A função estimada pode ser observada com a seguinte especificação:

$$
y_{t}=\sum_{i=1}^{h}\left[\alpha_{0}^{(i)}+\sum_{j=1}^{p} \alpha_{j}^{(i)} y_{t-j}+\sum_{j=1}^{q} \beta_{j}^{(i)} x_{j, t}+\varepsilon_{t}^{(i)}\right] I_{i}\left(q_{t}\right)
$$

Os autores concluem que o modelo não-linear é significativamente melhor do que o um com regra tipo Taylor ajustada para as taxas de juros brasileiras, o que mostra o duplo comportamento da função de reação do BACEN. Desse modo, o modelo não-linear é a maneira mais adequada, de acordo com o resultado desse estudo, para explicar a função de reação do BACEN entre agosto de 1994 e dezembro de 2000.

Soares e Barbosa (2006) procuram estimar uma regra de Taylor para o Brasil a partir da adoção do regime de metas de inflação em junho de 1999 considerando a taxa de juros real de equilíbrio de longo prazo da economia e a meta inflacionária variáveis no tempo. O modelo de estimação da função de reação proposta por esse trabalho pode ser observado na seguinte especificação:

$$
\begin{aligned}
& \Delta i_{t}=-\varphi\left(i_{t-1}-\pi^{*}-r_{t}^{*}\right)+\varphi \beta\left(\pi_{t+n}-\pi_{t}^{*}\right)+\varphi y_{1} x_{t} \\
& +\varphi y_{2} x_{t-1}+\varphi \lambda\left(\Delta x r_{t}-\Delta x r_{t-1}+\rho \Delta i_{t-1}\right.
\end{aligned}
$$

em que $\Delta i_{t}=$ primeira diferença da taxa de juros nominal, $\pi_{t+n}=$ a inflação no período $\mathrm{t}+\mathrm{n}, x_{t}=$ hiato do produto, $\pi_{t}^{*}=$ meta de inflação no ano $\mathrm{t}$, $i_{t-1}=$ taxa de juros defasada um período, $\Delta x r_{t}=$ variação da taxa de câmbio real, $\rho=$ coeficiente da primeira diferença da taxa de juros defasada, $\beta=$ coeficiente do diferencial da inflação e $\phi \in[0,1]$. Os autores baseiam seus modelos econométricos estimados em uma versão forward-looking da regra de Taylor, isto é, considera-se expectativa de inflação corrente ao invés da inflação corrente ou passada. O índice de preço utilizado para a medida da inflação é o IPCA calculado pelo IBGE. Soares e Barbosa (2006) concluíram 
que as evidências empíricas apresentadas não rejeitam a hipótese de que a taxa de juros real de equilíbrio de longo prazo varia ao longo do tempo. Os valores calculados do coeficiente de suavização sugerem que o BACEN ajusta a taxa básica de juros de maneira gradual. Em relação às estimativas do hiato da inflação, os autores afirmam que o BACEN, no período analisado, reagiu agressivamente ao desvio entre a expectativa de inflação e sua meta, a partir da adoção do regime de metas de inflação.

Policano e Bueno (2006) buscam estimar uma regra de política monetária através do método Time Varying Parameter (TVP), no qual é permitido que seus coeficientes variem em cada período de tempo de acordo com um processo de "passeio aleatório". Os resultados obtidos pelo modelo na realização de suas estimações, indicam que entre janeiro de 1995 e outubro de 2005, a política monetária brasileira se dividiu em dois períodos. O primeiro é associado ao regime de câmbio fixo, a taxa básica de juros reagiu negativamente às reservas internacionais e positivamente ao hiato do produto. No segundo período, caracterizado pela adoção do regime de metas para a inflação, a Selic respondeu principalmente às expectativas inflacionárias.

Lima et al. (2007) estimam a regra de política monetária adotada pelo BACEN no período pós-Plano Real. Com o intuito de lidar com a incerteza referente às datas das mudanças ocorridas nos parâmetros da função de reação, os autores adotaram a hipótese de que essas mudanças são regimes dependentes e a probabilidade de ocorrência desses regimes segue uma cadeia de Markov de primeira ordem. Desse modo, o modelo econométrico sugerido por esses autores possui uma estrutura flexível que permite detectar possíveis desvios em relação a uma função de reação linear simples. Esse modelo markoviano de regressão com modificação de regime é descrito de acordo com as seguintes equações:

$$
\begin{gathered}
y_{t}=a\left(S_{t}\right)+\sum_{m=1}^{p} b_{m}\left(S_{t}\right) y_{t-m}+\sum_{m=1}^{p} c_{m}\left(S_{t}\right) x_{t-m}+\varepsilon_{t} \\
\varepsilon_{t} \sim N\left(0, \sigma^{2}\left(S_{t}\right)\right) \\
P=\left[p_{i j}\right], \quad p_{i j}=\operatorname{Pr}\left[S_{t}=i \mid S_{t-1}=j\right], i, j=1, \ldots, 4
\end{gathered}
$$

em que $y_{t}$ é a taxa de juros nominal, $x_{t}$ é um vetor de variáveis que o Banco Central leva em consideração quando da fixação da taxa de juros, $S_{t}$ é uma variável aleatória que evolui de acordo com a matriz de transição apresentada na equação (9).

Portanto, esses autores concluem que de julho de 1996 a janeiro de 2006, a política monetária no Brasil pode ser caracterizada por quatro regimes, onde identificaram diferenças substanciais no modo como a política monetária foi conduzida nos períodos anterior e posterior a 1999, quando houve a passagem de câmbio administrado para flutuante. A política monetária no primeiro regime esteve preocupada com a defesa da taxa de câmbio e as mudanças nas 
taxas de juros se deveram fundamentalmente aos movimentos nas reservas internacionais. O segundo regime está associado a períodos de menor estresse no mercado de câmbio, o que permitiu ao Banco Central observar os valores assumidos pela inflação, nível de produção, e ainda a dinâmica da taxa de juros antes de ser definida a taxa Selic. Entre agosto de 1999 e janeiro de 2006 a política monetária brasileira, associada à adoção do regime de metas para inflação e de câmbio flutuante, foi caracterizada por outros dois estados recorrentes, uma vez que no terceiro regime a atenção do BACEN voltou-se à taxa de inflação, diminuindo a atenção dada à taxa de câmbio e ao produto. Em relação ao quarto regime, os autores concluem que o BACEN apresentou um comportamento mais flexível, dando atenção tanto à inflação como ao hiato do produto.

Barcellos Neto e Portugal (2007) com o objetivo de analisar a estabilidade da regra de política monetária do tipo Taylor (1993) no Brasil após a implementação do regime de metas para a inflação e tendo em conta a mudança na presidência do Banco Central em 2003, partem de uma função de reação dada por:

$$
\begin{aligned}
& i_{t}=\beta_{1} i_{t-j}+\beta_{2} D j_{t}+\beta_{3} y_{t-2}+\beta_{4} c_{t-1}+\beta D_{1} D j_{t} \\
& +\beta_{6} D_{1} y_{t-2}+\beta_{7} D_{1} c_{t-1}+\beta_{8} \text { Dout } 2002+\varepsilon_{t}
\end{aligned}
$$

onde $i_{t}=$ taxa Selic mensal (média do período); $D j_{t}=$ desvio das expectativas de inflação em relação à meta; D1 = variável dummy que assume valor igual a 1 para o período de janeiro de 2003 a dezembro de 2006 e um valor zero, caso contrário (janeiro de 2000 a dezembro de 2002); e Dout2002 = variável dummy com valor unitário em outubro de 2002 e zero no restante dos meses. Os resultados obtidos com as estimativas realizadas por esses autores foram os de que existe uma forte evidência da continuidade e do compromisso com o sistema de metas no Brasil desde sua implementação.

Isso pôde ser observado quando o Banco Central esteve sob a gestão tanto de Armínio Fraga quanto de Henrique Meirelles. Embora tenha se mantido uma estrutura de regra monetária do tipo Taylor (1993), algumas mudanças foram detectadas, dentre as quais se destacam: i) a taxa Selic na gestão de Meirelles reagiu 3,3 vezes menos em relação aos desvios da inflação esperada em relação à meta inflacionária em comparação à gestão Fraga; ii) o comportamento da taxa Selic em resposta as variações cambiais foi mais significativa durante a administração de Henrique Meirelles do que na administração de Armínio Fraga.

Aragón e Portugal (2010) buscaram evidenciar a existência de assimetrias nas preferências do Banco Central do Brasil através da realização de testes de quebras estruturais para especificações da função de reação que são nãolineares em decorrência de preferências assimétricas do Banco Central em relação a desvios positivos e negativos da inflação em relação à meta e/ou do 
produto em relação ao produto potencial.

Oliveira e Aragón (2011) buscaram evidenciar a ocorrência de mudança estrutural na regra de política monetária adotada pelo Banco Central do Brasil no período de 2000 a 2009, para alcançar tal utilizaram as metodologias propostas por Chow (1960), Andrews (1993) e Andrews e Ploberger (1994), este último consiste estimar a ocorrência de um ponto de quebra estrutural em uma data desconhecida. Dessa forma, os autores concluíram que desde adoção do regime de metas inflacionárias os coeficientes da regra de política monetária adotada pelo BACEN durante o período de 2000-2009 permaneceram constantes. Esse resultado diferiu das evidências em favor de mudanças estruturais na função de reação do Banco Central encontradas por Lima et al. (2007) e Barcellos Neto e Portugal (2007).

Medeiros e Aragón (2011) inovam em relação ao trabalho elaborado por Aragón e Portugal (2010) ao utilizarem a metodologia proposta por Bai e Perron (1998, 2003) e realizam testes de quebra estruturais para funções de reação nãolineares e ao considerar, através de uma extensão do modelo teórico proposto por Surico (2007), as preferências do policymaker assimétricas e tomam como base um modelo macroeconômico de uma pequena economia aberta, obtendo assim, uma regra de política monetária ótima para a autoridade monetária. Neste sentido, os autores concluem que há possíveis alterações no modo de condução da política monetária , assim como nos parâmetros de assimetrias das preferências do BACEN e que geralmente, os coeficientes das funções de reação estimadas apontam para o fato de que o BACEN reagiu de maneira mais forte a desvios da inflação, tanto corrente como a esperada, em relação à meta $\mathrm{e}$ ao hiato do produto depois do ano de 2003.

\subsection{A Política Monetária no Brasil Pós-99}

Com a implementação do Plano Real em 1994, a condução da política monetária foi dividida em dois períodos. De julho de 1994 a janeiro de 1999, no qual o procedimento de estabilização de preços foi caracterizado pelo estabelecimento de uma política de bandas cambiais. A segunda fase da condução da política monetária de estabilidade dos preços no Plano Real foi inaugurada em janeiro de 1999, como complemento de transição, logo após a mudança do regime cambial, com a introdução da livre flexibilidade. Posteriormente a adoção do regime de câmbio flutuante e devido ao cenário de fortes pressões inflacionárias, foi instituído como estratégia de condução da política monetária brasileira o regime de metas para inflação através do Decreto $\mathrm{n}^{\mathrm{o}}$. 3.088, de 21 de junho do mesmo ano.

A implantação do regime de metas apara a inflação foi motivada, sobretudo, pelo anseio de recuperação da credibilidade da política monetária, dado que os agentes tinham dúvidas quanto à capacidade do governo em controlar o 
processo inflacionário depois do abandono do regime de âncora cambial. Outro motivo se deveu a conclusão a que chegou o Comitê de Política Monetária (Copom), de que em um regime de câmbio flutuante não é possível o controle da taxa de inflação via intervenção na taxa de câmbio, uma vez que o valor do dólar era determinado no mercado.

Assim, com a adoção de metas inflacionárias, a condução da política monetária foi voltada para o cumprimento de uma meta explícita de inflação com intervalos de tolerância definidos pelo Conselho Monetário Nacional (CMN). A atuação da autoridade monetária passava a ajustar-se pelo compromisso de controle da inflação, passando o Banco Central do Brasil a ter, em prática, certa independência operacional, isto é, liberdade para utilizar instrumentos de política monetária de modo a alcançar a meta de inflação estabelecida.

No entanto, ficou a cargo do governo a definição das metas e das bandas de variação para a inflação. O Índice de Preços ao Consumidor Amplo (IPCA), calculado pelo Instituto Brasileiro de Geografia e Estatística (IBGE), passou a ser o índice de preços que baliza o regime monetário.

O Banco Central utiliza como principal instrumento de política a definição de uma meta anual da taxa básica de juros, a taxa Selic, para garantir a convergência da inflação para a meta anunciada, ficando as variações dessa condicionadas ao controle do processo inflacionário.

Vale destacar que se as metas não forem atingidas, ou seja, se a inflação não se situar dentro dos limites pré-estabelecidos, de acordo com o decreto $\mathrm{n}^{0} .3 .088$, cabe ao presidente do Banco Central divulgar, em carta aberta ao Ministro da Fazenda, os motivos do descumprimento bem como as providências e prazo para o retorno da taxa de inflação aos limites estabelecidos. Com a adoção desse arcabouço de política monetária, fica claro que o governo tem como sua principal preocupação, o compromisso com a estabilidade de preços da economia.

TABELA 1. METAS DE INFLAÇÃO NO BRASIL: 1999 - 2011

\begin{tabular}{|c|c|c|c|c|c|}
\hline ANO & NORMA & $\begin{array}{c}\text { META } \\
(\%)\end{array}$ & $\begin{array}{c}\text { BANDA } \\
\text { (P.P.) }\end{array}$ & $\begin{array}{c}\text { LIMITES } \\
\text { INFERIOR E } \\
\text { SUPERIOR (\%) }\end{array}$ & $\begin{array}{c}\text { INFLAÇÃO } \\
\text { EFETIVA } \\
\text { (IPCA \% } \\
\text { A.A.) }\end{array}$ \\
\hline 1999 & & 8 & 2 & $6-10$ & 8,94 \\
\hline 2000 & RESOLUÇÃO 2.615 & 6 & 2 & $4-8$ & 5,97 \\
\hline 2001 & & 4 & 2 & $2-6$ & 7,67 \\
\hline 2002 & RESOLUÇÃO 2.744 & 3,5 & 2 & $1,5-5,5$ & 12,53 \\
\hline \multirow{2}{*}{$2003^{1 /}$} & RESOLUÇÃO 2.842 & 3,25 & 2 & $1,25-5,25$ & \\
\hline & RESOLUÇÃO 2.972 & 4 & 2,5 & $1,5-6,5$ & 9,30 \\
\hline
\end{tabular}




\begin{tabular}{llcccc}
\hline \multirow{2}{*}{$2004^{1 /}$} & RESOLUÇÃO 2.972 & 3,75 & 2,5 & $1,25-6,25$ & \\
& RESOLUÇÃO 3.108 & 5,5 & 2,5 & $3-8$ & 7,60 \\
\hline 2005 & RESOLUÇÃO 3.108 & 4,5 & 2,5 & $2-7$ & 5,69 \\
\hline 2006 & RESOLUÇÃO 3.210 & 4,5 & 2 & $2,5-6,5$ & 3,14 \\
\hline 2007 & RESOLUÇÃO 3.291 & 4,5 & 2 & $2,5-6,5$ & 4,46 \\
\hline 2008 & RESOLUÇÃO 3.378 & 4,5 & 2 & $2,5-6,5$ & 5,90 \\
\hline 2009 & RESOLUÇÃO 3.463 & 4,5 & 2 & $2,5-6,5$ & 4,31 \\
\hline 2010 & RESOLUÇÃO 3.584 & 4,5 & 2 & $2,5-6,5$ & 5,91 \\
\hline 2011 & RESOLUÇÃO 3.748 & 4,5 & 2 & $2,5-6,5$ & 6,50 \\
\hline FONTE:
\end{tabular}

FONTE: Banco Central.

A Tabela 1 acima mostra as metas de inflação para o Brasil no período de 1999 a 2009, nela observa-se que os dois primeiros anos do regime de metas de inflação, assim como os anos de 2004, 2005, 2006, 2007, 2008, 2009, 2010 e 2011 a política monetária apresentou efeito na convergência da inflação à meta estabelecida, com estaque para os anos de 2004, 2006, 2007 e 2009, onde a inflação efetiva situou-se abaixo do centro da meta preestabelecida pela autoridade monetária e para o ano de 2011, onde a inflação atingiu o limite superior do centro da meta preestabelecida.

FIGURA 1. INFLAÇÃO NO BRASIL: 1999-2011

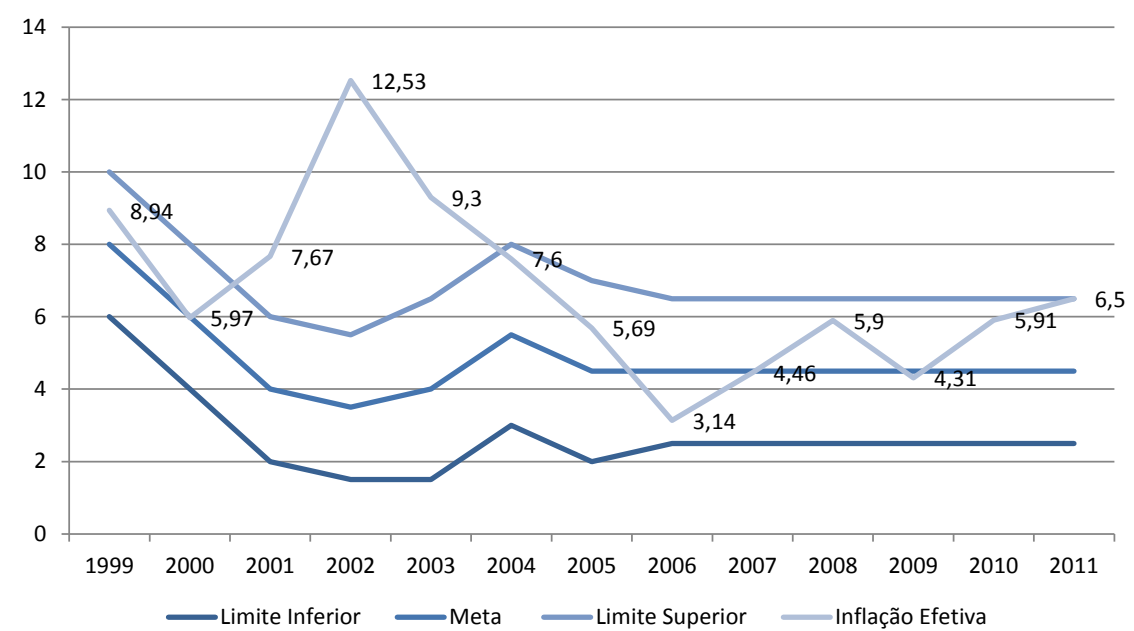

Fonte: Banco Central.

Desde a implementação do regime de metas para a inflação no Brasil em 1999, a atuação do BACEN e a política monetária proporcionaram alternâncias em relação à eficácia em convergir à meta estipulada, como pode ser observado 
na Figura 1. Foi a partir de 2004, que o nível de preços da economia nacional obedeceu a rigor as metas estabelecidas pelo $\mathrm{CMN}$, mostrando progressivamente índices relativamente menores, atingindo índices abaixo do centro da meta, como no ano de 2006, e bem próximo a ele, porém abaixo, como em 2007 e 2009. Mesmo tendo sofrido aumentos relativos à taxa de inflação nos anos de 2007 e 2008 se manteve dentro do intervalo de tolerância aceito para as metas inflacionárias. Em 2009, observa-se a retomada da trajetória relativamente descendente dessa taxa, destacando-se também o ano de 2011, onde a inflação atingiu o limite superior do centro da meta preestabelecida.

\section{Estratégia Empírica}

\subsection{Procedimentos econométricos}

De acordo com Greene (2003), quando se emprega um modelo de regressão que envolve o uso de séries temporais, pode-se observar uma mudança estrutural na relação entre o regressando e os regressores. Por mudança estrutural se entende que os valores dos parâmetros do modelo não se mantêm iguais durante todo o período considerado. As possíveis diferenças, isto é, as prováveis mudanças estruturais, podem ocorrer por meio de diferenças no intercepto ou no coeficiente angular, ou em ambos.

Para verificar a presença de mudança estrutural, utilizamos os testes de Bai e Perron (2003), onde a data da quebra estrutural é suposta desconhecida.

\subsubsection{O Modelo de Múltiplas Quebras Estruturais}

Para verificar possíveis alterações nos valores dos parâmetros de um modelo, pode-se utilizar o teste de quebra estrutural proposto por Bai e Perron (2003) que propõem uma formulação para a obtenção endógena de múltiplas mudanças estruturais, considerando em princípio a seguinte regressão múltipla linear com $m$ mudanças estruturais:

$$
y_{t}=x_{t} \beta+z_{t} \delta_{j}+u_{t} \quad t=T_{j-1}+1, \ldots, T_{j}
$$

para $\mathrm{j}=1, \ldots, \mathrm{m}+1$.

em que: $y_{t}$ é observada como a variável dependente do tempo t; $x_{t}$ e $z_{t}$ são vetores de variáveis independentes de ordem ( $\mathrm{p} \times 1)$ e (q x 1), respectivamente; $\beta$ e $\delta_{j}$ são os vetores dos coeficientes que correspondem a $x_{t}$ e $z_{t}$ e $u_{t}$ é o termo de erro. Os índices $\left(T_{1}, \ldots, T_{m}\right)$ correspondem aos pontos de quebra e dados como desconhecidos, ao se fazer uso da convenção em que $\left(T_{0}=0 \mathrm{e}\right.$ 
$T_{m+1}=T$ ).

A especificação (11) pode ser representada em forma de uma matriz:

$$
Y=X \beta+\bar{Z} \delta+U
$$

em que :

$Y=\left(y_{1}, \ldots, y_{T}\right), X=\left(x_{1}, \ldots, x_{T}\right), U=\left(u_{1}, \ldots, u_{T}\right), \delta=\left(\delta_{1}, \delta_{2}, \ldots, \delta_{m+1}\right)$ e $\bar{Z}$ é a matriz de partições diagonais de $Z$ em $\left(T_{1}, \ldots, T_{m}\right)$, ou seja, $\bar{Z}=\operatorname{diag}\left(Z_{1}, \ldots, Z_{m+1}\right) \operatorname{com} Z_{i}=\left(Z_{T_{i-1}}+1, \ldots, Z_{T}\right)$.

Dado que a estimação considerada é baseada no princípio do MQO (Mínimos Quadrados Ordinários) o método de estimação proposto baseia-se em três passos fundamentais:

Para cada m-participação $\left(T_{1}, \ldots, T_{m}\right)$, as estimativas por MQO $\beta$ e $\delta_{j}$ dos vetores são alcançadas através da minimização da soma dos quadrados dos resíduos:

$$
(Y-X \beta-\bar{Z} \delta)^{\prime}(Y-X \beta-\bar{Z} \delta)=\sum_{i=1}^{m+1} \sum_{t=T_{i-1}+1}\left[y_{t}-x_{t}^{\prime} \beta-z_{t}^{\prime} \delta_{i}\right]^{2}
$$

Sendo $\hat{\beta}\left(\left\{T_{j}\right\}\right)$ e $\hat{\delta}\left(\left\{T_{j}\right\}\right)$ as estimativas fundamentadas sob uma dada m-participação $\left(T_{1}, \ldots, T_{m}\right)$ denominada por $\left\{T_{j}\right\}$. Ao se substituir na função objetivo (13) e designando a soma dos quadrados dos resíduos resultante como $S_{t}\left(T_{1}, \ldots, T_{m}\right)$ os pontos de quebra $\left(\hat{T}_{1}, \ldots, \hat{T}_{m}\right)$ são representados pela seguinte especificação:

$$
\left(\hat{T}_{1}, \ldots, \hat{T}_{m}\right)=\arg \min _{\left\{T_{j}\right\}} S_{T}\left(T_{1}, \ldots, T_{m}\right)
$$

em que a minimização é adotada em todas as participações $\left(T_{1}, \ldots, T_{m}\right)$, tais que $T_{i}-T_{i-1} \geq q$.

Neste sentido, as estimações dos pontos de quebra implicam em mínimos globais da função objetivo.

Finalmente, como último passo tem-se que a estimação dos parâmetros da regressão são estimativas unificadas com a participação $\mathrm{m}\left\{T_{j}\right\}$ sendo produzidas por $\hat{\beta}\left(\left\{T_{j}\right\}\right)$ e $\hat{\delta}\left(\left\{T_{j}\right\}\right)$.

3.1.2. Metodologia para o cálculo de mínimos globais

Objetivando obter as estimativas dos pontos de quebra, Bai e Perron (2003) ponderaram um algoritmo fundamentado no princípio da programação 
dinâmica que torna possível estimar pontos como minimizadores globais da soma dos quadrados dos resíduos. Vale salientar que este algoritmo usa, no máximo, operações de MQO de ordem $O\left(T^{2}\right)$ seja qual for o número de mudanças estruturais.

\subsubsection{Modelo de mudança estrutural puro}

Na presente proposta será considerado um modelo de mudança estrutural puro, que é dado por:

$$
Y=\bar{Z} \delta+U
$$

Neste caso, o cálculo das estimativas $\hat{\delta}, \hat{u}_{t}$ e $S_{t}\left(T_{1}, \ldots, T_{m}\right)$ pode ser realizado aplicando-se MQO para cada sub-amostra relevante. Para cada segmento relevante, o cálculo da soma do quadrado dos resíduos pode ser alcançado por meio do uso de fórmulas de atualização padrão para cálculo de resíduos recursivos.

Na realidade, pode-se calcular a partir de $T-h m+1$ conjunto de resíduos, em que h corresponde a alguma distância mínima entre cada quebra, todas as informações pertinentes. Uma vez que $v(i, j)$ é o resíduo recursivo no instante $\mathrm{j}$ obtido através do uso de uma amostra que se inicia no dia $\mathrm{i}$, e sendo $\operatorname{SSR}(i, j)$ a soma do quadrado dos resíduos originados por meio da aplicação dos mínimos quadrados de um segmento que tem início na data i e termina da data $\mathrm{j}$, desse modo, a seguinte relação recursiva é dada como $\operatorname{SSR}(i, j)=\operatorname{SSR}(i, j-1)+v(i, j)^{2}$. Uma vez que o conjunto de todas as informações está contido nos valores de $\operatorname{SSR}(i, j)$ para as combinações (i,j). Portanto, é necessário um número de matriz inversa de ordem $O(T)$.

\subsubsection{Algoritmo de programação dinâmica}

Com o intuito de estimar de maneira mais eficiente o modelo de múltiplas quebras estruturais aqui sugerido, pretende-se utilizar o algoritmo da programação dinâmica proposto no trabalho de Bai e Perron (2003).

De acordo com o exame proposto por Bai-Perron (1998, 2003), as rupturas estruturais a serem identificadas são aquelas demarcadoras de períodos cujas somas dos quadrados dos resíduos (SSR) das regressões de MQO se constituam localmente mínimas, dado que se confronta às SSR de todos os demais períodos possíveis.

Levando em consideração que a soma dos quadrados dos resíduos de relevantes segmentos tenham sido calculados e guardados, o enfoque de programação dinâmica pode ser utilizado para estimar qual partição 
alcança uma minimização global da soma dos quadrados dos resíduos. Esta metodologia parte, fundamentalmente, de um exame sequencial de partições ótima de uma quebra, ou dois segmentos. Neste sentido, sendo $\operatorname{SRR}\left(\left\{T_{n, r}\right\}\right)$ a soma dos quadrados residuais agregados à partição ótima contendo r rupturas usando as primeiras n observações. Assim, a partição ótima é capaz de resolver o seguinte problema recursivo:

$$
\operatorname{SRR}\left(\left\{T_{m, T}\right\}\right)=\min _{m h \leq j \leq T-h}\left[\operatorname{SRR}\left(\left\{T_{m-1, j}\right\}\right)+\operatorname{SSR}(j+1, T)\right]
$$

\subsection{O modelo empírico}

O presente trabalho busca estimar uma função de reação do Banco Central do Brasil, tendo por base a estrutura proposta por Taylor (1993). Através da leitura das atas das reuniões do Comitê de Política Monetária (COPOM) foi possível identificar as variáveis que normalmente são utilizadas pelo BACEN na determinação de sua regra monetária. Dado que o ambiente de atuação operacional dos bancos centrais é de bastante incerteza, a autoridade monetária tende a suavizar sua política via variações progressivas em seu instrumento de política monetária, a taxa básica de juros. Posto isto, a especificação para a função de reação do BACEN que procuraremos estimar é dada por:

$$
i_{t}=\beta_{0, j}+\beta_{1, j} D j_{t}+\beta_{2, j} \operatorname{Gap}_{t-2}+\beta_{3, j} \Delta e_{t-1}+\rho_{1, j} i_{t-1}+\rho_{2, j} i_{t-2}+\varepsilon_{t}
$$

para $j=1,2$

em que $i_{t}$ é a taxa Selic mensal (média do período) no período t, $D j_{t}$ é a média ponderada entre os desvios no ano corrente e no ano seguinte da expectativa da inflação em relação a sua meta de cada ano, $G a p_{t-2}$ é a diferença do produto efetivo em relação ao seu potencial defasado em dois períodos, $\Delta e_{t-1}$ é a variação da taxa de câmbio nominal (R\$/US\$) no período t-1 e $\varepsilon_{t}$ é o termo de erro.

Desse modo, o comportamento da autoridade monetária quanto à definição da taxa básica de juros nominal de curto prazo leva em consideração a ponderação dos desvios das expectativas em relação às metas no ano corrente e no ano seguinte, a diferença percentual defasada em dois períodos do produto efetivo em relação ao produto potencial, a variação da taxa de câmbio nominal defasada em um período e as taxas de juros nominais defasadas em um e dois períodos. O efeito imediato das variáveis explicativas $D j_{t}, G a p_{t-2}$ e $\Delta e_{t-1}$ sobre a taxa de juros de curto prazo é representado pelos coeficientes $\beta_{1}, \beta_{2}$ e $\beta_{3}$, enquanto que $\rho_{1}$ e $\rho_{2}$ medem a sensibilidade da taxa Selic em resposta as variações nos componentes inerciais $i_{t-1}$ e $i_{t-2}$, respectivamente.

Entretanto, ao determinar ou reajustar a taxa básica de juros da economia 
para o curto prazo a autoridade monetária leva em consideração os efeitos que essas medidas podem causar sobre a taxa de juros futura. Dito de outra forma, o Banco Central do Brasil considera os efeitos secundários causados pelas variáveis explicativas na taxa Selic. Isso pode ser observado através do componente inercial $i_{t-1} \mathrm{e} i_{t-2}$ que compõem a regra monetária de curto prazo, isto é, a regra no período t irá influenciar as decisões do BACEN quanto à determinação da taxa básica de juros nos períodos $t+1$ e $t+2$. Assim, podemos medir esses efeitos secundários através da expressão da regra de política monetária de longo prazo, dada por:

$$
i=\gamma_{0}+\gamma_{1} D j_{t}+\gamma_{2} \operatorname{Gap}_{t-2}+\gamma_{3} \Delta e_{t-2}+\varepsilon_{t}
$$

onde $\gamma_{i}=\frac{\beta_{i}}{1-\rho_{1}-\rho_{2}}$, para $\mathrm{i}=0,1,2,3$, representam a resposta de longo prazo da taxa Selic as variáveis explicativas $D j_{t}, \operatorname{Gap}_{t-2}$ e $\Delta e_{t-1}$.

\section{Descrição dos dados e testes de raiz unitária}

Foram utilizados dados com periodicidade mensal que compreenderam o período de janeiro de 2000 a dezembro de 2011, coletados dos sites de pesquisa do Instituto de Economia Aplicada (IPEA) e do Banco Central do Brasil (BACEN).

A taxa de juros $\left(i_{t}\right)$ utilizada será a taxa Selic acumulada no mês e anualizada. Para medir a sensibilidade da taxa de juros em relação à diferença entre a inflação e sua meta, utilizaremos a variável $D j$, proposta em Minella et al. (2003). Essa variável é estabelecida a partir das metas de inflação decididas em reunião prévia do Copom para os anos T e $\mathrm{T}+1$ e da pesquisa diária que o Banco Central realiza entre as instituições financeiras e as firmas de consultoria, onde são coletados os valores que esses agentes econômicos esperam para a inflação no período T $\left(E_{j} \pi_{T}\right)$ e $\mathrm{T}+1\left(E_{j} \pi_{T+1}\right)$. Assim sendo, a variável $D j$ assume a seguinte especificação:

$$
D j_{t}=\frac{(12-j)}{12}\left(E_{j} \pi_{T}-\pi_{T}^{*}\right)+\frac{j}{12}\left(E_{j} \pi_{T+1}-\pi_{T+1}^{*}\right)
$$

O hiato do produto é medido pela diferença percentual entre o índice de produção industrial dessazonalizado $\left(y_{t}\right)$ e o produto potencial $\left(y p_{t}\right)$, e é dado por:

$$
\text { gap }=100 \frac{\left(y_{t}-y p_{t}\right)}{y p_{t}}
$$

Também utilizamos como proxy para o produto potencial o produto estimada 
pelo filtro Hodrick-Prescott (HP)1.

Por fim, para variável taxa de câmbio $\left(e_{t}\right)$ empregamos a média da taxa de câmbio comercial para venda (R \$/US\$) com periodicidade mensal.

A primeira etapa a ser tomada quando se trabalha com uma série temporal é verificar se esta é produzida por um processo estocástico estacionário, ou seja, se as suas média e variância se apresentam constantes ao longo do tempo e o valor da covariância entre dois períodos de tempo depende apenas da distância entre os dois períodos. A importância desta análise relaciona-se ao fato de que, em modelos econométricos que incluem variáveis não-estacionárias, relações espúrias (ou ilegítimas) que podem aparecer na regressão obtida, fazendo com que os testes t e F usuais não sejam válidos se estas variáveis não apresentarem uma relação de co-integração.2

Com a finalidade de verificar se as variáveis utilizadas em nosso modelo seguem um processo estocástico estacionário, realizamos três testes, a saber: ADF (Augmented Dickey-Fuller); Phillips-Perron (PP); KPSS, proposto por Kwiatkowski et al. (1992).

Os testes de raiz unitária $\mathrm{ADF}$ e $\mathrm{PP}$ têm como hipótese nula que a série é nãoestacionária (ou raiz unitária), ao passo que o teste KPSS testa a hipótese nula de que a série é estacionária. Com base no critério de informação de Akaike (AIC) foi selecionado um número ótimo de termos de diferença defasados a serem incluídos em cada regressão ( $\mathrm{k}$ ) levando em conta um número máximo de defasagens de $k \max =\operatorname{int}(12(\mathrm{~T} / 100) 1 / 4)=13$. Ademais, introduziu-se como elementos determinísticos a constante (c) e uma tendência linear (t) no caso em que esses componentes se mostraram estatisticamente significativos. Neste sentido, os resultados dos testes apresentados na Tabela 2 apontam que as variáveis estimadas no modelo podem ser consideradas estacionárias.

TABELA 2. TESTE DE RAIZ UNITÁRIA

\begin{tabular}{ccccc}
\hline VARIÁVEL & $\begin{array}{c}\text { REGRESSORES } \\
\text { EXÓGENOS }\end{array}$ & ADF & PP & KPSS \\
\hline$I_{T}$ & $\mathrm{C}, \mathrm{T}$ & $-4,231^{*}(1)$ & $-2,62 \mathrm{O}^{\mathrm{N} . \mathrm{S}}$ & $0,136^{* * * *}$ \\
$D J_{T}$ & $\mathrm{C}$ & $-2,789^{* * * *}(2)$ & $-2,903^{* * *}$ & $0,322^{\mathrm{N} . \mathrm{S}}$ \\
$G A P_{T}$ & - & $-3,975^{* *}(1)$ & $-3,699^{*}$ & $0,030^{\mathrm{N} . S}$ \\
$\Delta \mathrm{E}_{T}$ & - & $-7,752^{*}(0)$ & $-7,754^{*}$ & $0,141^{\mathrm{N.S}}$ \\
\hline
\end{tabular}

Nota: * Significativo a $1 \% .{ }^{* *}$ Significativo a $5 \% .{ }^{* *}$ Significativo a 10\%. n.s Não- significativo. 


\section{Teste de quebras estruturais}

Para identificar possíveis mudanças nos valores dos parâmetros na regra de política monetária do Banco Central do Brasil no período de 2000 a 2011, realizamos o teste de Bai e Perron $(1998,2003)$ que permite observar a ocorrência de múltiplas quebras estruturais. O número máximo de quebras fixados nesse teste foi de dois com uma aparagem de $15 \%(\varepsilon=0.15)$.

TABELA 3. TESTE DE BAI E PERRON PARA QUEBRA ESTRUTURAL

\begin{tabular}{ccccccc}
\hline \multicolumn{3}{c}{$\operatorname{SupF}_{T}(1)$} & $\operatorname{SupF}_{T}(2)$ & \multicolumn{3}{c}{$\operatorname{SupF}_{T}(2 / 1)$} \\
\hline \multicolumn{3}{c}{28,16} & \multicolumn{5}{c}{28,84} & 39,57 \\
$10 \%$ & & \multicolumn{5}{c}{ VALORES CRÍTICOS } \\
$5 \%$ & 17,97 & $10 \%$ & 16,02 & $10 \%$ & 20,01 \\
$1 \%$ & 20,08 & $5 \%$ & 17,37 & $5 \%$ & 22,11 \\
\hline
\end{tabular}

Fonte: elaboração própria.

A Tabela 3 acima mostra os resultados do teste de quebra estrutural. Para testar a hipótese nula de ausência de quebra $(\mathrm{m}=0)$ contra a hipótese alternativa $(m=1)$ e $(m=2)$ quebras são utilizadas as estatísticas $\operatorname{SupF}_{T}(1)$ e $\operatorname{Sup}_{T}(2)$ , respectivamente. Nos dois casos, a hipótese de constância nos parâmetros da função de reação estimada é rejeitada a um nível de significância de 10\%. A hipótese nula de que há uma quebra versus a alternativa de duas quebras estruturais para o teste $\operatorname{SupF}_{T}(2 / 1)$ também é rejeitada ao nível de $10 \%$.

\subsection{Estimação da função de reação para o BACEN (2000-2011)}

Dado que o teste de quebra estrutural de Bai e Perron $(1998,2003)$ indica uma mudança comportamental nas decisões de política monetária por parte da autoridade monetária brasileira desde a adoção do regime de metas para inflação. Para melhor visualização dessas mudanças, são estimadas função de reação para os períodos antes e depois das quebras estruturais. As datas de quebra estimadas pelo teste de quebra estrutural de Bai e Perron (2003) foram fevereiro de 2004 e outubro de 2007.

Podemos observar na Tabela 4 os resultados da estimação da regra monetária do BACEN. No entanto, vale destacar que para corrigir os problemas de autocorrelação e heterocedasticidade nos resíduos do modelo, as funções de reação foram estimadas com a matriz HAC (Newey-West). 
TABELA 4. ESTIMAÇÕES DAS FUNÇÕES DE REAÇÃO PARA O BACEN: 2000:01 $-2011: 12$

\begin{tabular}{|c|c|c|c|c|}
\hline PARÂMETROS & 00:01/12:05 & 00:01/04:02 & 04:03/07:10 & 07:02/12:05 \\
\hline$\beta_{0}$ & $\begin{array}{c}0.27^{*} \\
(0.053)\end{array}$ & $\begin{array}{c}2.10^{*} \\
(0.003)\end{array}$ & $\begin{array}{c}0.48^{*} \\
(0.004)\end{array}$ & $\begin{array}{c}0.28^{*} \\
(0.074)\end{array}$ \\
\hline$\beta_{1}$ & $\begin{array}{l}0.1300^{*} \\
(0.005)\end{array}$ & $\begin{array}{l}0.3266^{*} \\
(0.000)\end{array}$ & $\begin{array}{l}0.1733^{*} \\
(0.003)\end{array}$ & $\begin{array}{l}0.0491^{*} \\
(0.080)\end{array}$ \\
\hline$\beta_{2}$ & $\begin{array}{l}0.0269^{*} \\
(0.000)\end{array}$ & $\begin{array}{c}-0.0026^{\mathrm{NS}} \\
(0.944)\end{array}$ & $\begin{array}{c}-0.0039^{\mathrm{NS}} \\
(0.758)\end{array}$ & $\begin{array}{l}0.0211^{*} \\
(0.016)\end{array}$ \\
\hline$\beta_{3}$ & $\begin{array}{c}0.0039^{\mathrm{NS}} \\
(0.676)\end{array}$ & $\begin{array}{c}0.0191^{\mathrm{NS}} \\
(0.312)\end{array}$ & $\begin{array}{c}-0.0122^{\mathrm{NS}} \\
(0.113)\end{array}$ & $\begin{array}{c}-0.0047^{\mathrm{NS}} \\
(0.455)\end{array}$ \\
\hline$\rho_{1}$ & $\begin{array}{l}1.6482^{*} \\
(0.000)\end{array}$ & $\begin{array}{l}1.4127^{*} \\
(0.000)\end{array}$ & $\begin{array}{l}1.7483^{*} \\
(0.000)\end{array}$ & $\begin{array}{l}1.6277^{*} \\
(0.000)\end{array}$ \\
\hline$\rho_{2}$ & $\begin{array}{l}-0.6739^{*} \\
(0.000)\end{array}$ & $\begin{array}{l}-0.5474^{*} \\
(0.000)\end{array}$ & $\begin{array}{c}-0.7828^{*} \\
(0.000)\end{array}$ & $\begin{array}{l}-0.6577^{*} \\
(0.000)\end{array}$ \\
\hline$R^{2}-$ ajustado & 0.995 & 0.985 & 0.997 & 0.984 \\
\hline
\end{tabular}

Nota: ${ }^{*}$ Significativo a $1 \% .{ }^{* *}$ Significativo a $5 \% .{ }^{* * *}$ Significativo a 10\%. NS=Não-significativo. Valores entre parênteses correspondem ao erro-padrão.

O valor do $\mathrm{R}^{2}$ - ajustado = 0,995 indica um excelente grau de explicação das variáveis independentes para o modelo. $\mathrm{Na}$ análise da resposta da taxa Selic em relação aos coeficientes de curto prazo, com exceção do coeficiente da variação da taxa de câmbio para o período t-1 $\left(\beta_{3}\right)$ que não foi significativo, todos os demais coeficientes apresentaram significância estatística de 1\%. Os valores dos coeficientes da variável $D j, \beta_{1}$, indicam que a postura de política monetária adotada pelo BACEN para o período considerado foi relativamente forte no sentido de controle inflacionário. Os baixos valores dos coeficientes $\beta_{2}$ para o gap do produto mostram que essa variável possui um impacto relativamente baixo na determinação da taxa básica de juros no período analisado. Além disso, nos intervalos em que ocorreram as quebras esses parâmetros foram não significantes. Os coeficientes $\beta_{3}$ da variação da taxa de câmbio nominal não se mostraram significativos no período analisado.

Neste sentido, os valores apresentados por esses coeficientes indicam que a autoridade monetária reage positivamente aos desvios da expectativa de 
inflação em relação a sua meta e ao gap do produto, sendo essa reação mais sensível aos desvios das expectativas inflacionárias em relação à meta de inflação. Vale ressaltar que aumento mais que proporcional da taxa de juros em resposta a um desvio da inflação em relação à meta mostra que esta regra de política satisfaz o princípio de Taylor (1993). Assim, quando há um aumento sustentado da inflação, o Banco Central aumenta a taxa de juros nominal em um valor suficiente para que a taxa real de juros se eleve, o gap produto se reduza e a inflação volte à sua meta.

\section{Considerações finais}

Como destacado na introdução, o presente trabalho buscou testar a hipótese da ocorrência de possíveis pontos de mudanças estruturais, isto é, de alteração na dinâmica de definição da taxa Selic pelo Banco Central brasileiro no período de metas de inflação. Para tal, utilizamos a metodologia proposta por Bai e Perron (2003).

Os resultados obtidos nesse teste de mudanças estruturais indicaram que há evidências de duas quebras estruturais nos coeficientes da função de reação do Banco central do Brasil no período de metas para a inflação, as datas das mudanças estruturais obtidas foram 2004.2 e 2007.10.

Os resultados das estimações mostraram que a postura de política monetária adotada pelo BACEN para o período considerado foi relativamente forte no sentido de controlar a inflação. Também constatou-se que a autoridade monetária brasileira tem reagido positivamente aos desvios da expectativa de inflação em relação a sua meta e ao gap do produto, sendo mais sensível aos desvios das expectativas inflacionárias em relação à meta de inflação.

Para trabalhos futuros, sugere-se que sejam realizados testes de quebras estruturais para especificações da função de reação que são não-lineares em decorrência de preferências assimétricas do Banco Central em relação a desvios positivos e negativos da inflação em relação a meta e/ou do produto em relação ao produto potencial (ver, por exemplo, Aragón e Portugal, 2010).

\section{Referências}

ANDREWS, D. W. Tests for parameter instability and structural change with unknown change point. Econometrica, v. 61, n. 4, p. 1383-1414, 07/1993.

ANDREWS, D. W.; PLOBERGER, W. Optimal tests when a nuisance parameter is present only under the alternative. Econometrica, v. 62, n. 6, p. 1383$141411 / 1994$.

ARAGÓN, E. K. da S. B.; PORTUGAL, M. S. Nonlinearities in Central Bank of Brazil's reaction function: the case of asymmetric preferences. Estudos Econômicos, v.40, n.2, 2010. 
BAI, J.; PERRON, P. Estimating and Testing Linear Models with Multiple Structural Changes. Econometrica, v. 66, p. 47-78, 1998.

BAI, J.; PERRON, P. Computation and analysis of multiple structural change models. Jornal of Applied Econometrics, v. 18, p. 1-22, Jan./Feb. 2003.

BANCO CENTRAL DO BRASIL. Relatório de Inflação. Vários números. Disponível em: < http://www.bacen.gov.br >. Acesso em: o9 de março de 2012.

BANCO CENTRAL DO BRASIL. Relatório de Inflação. Setembro de 1999. Disponível em: < http://www.bacen.gov.br >. Acesso em: o9 de março de 2012.

. Atas das Reuniões do Comitê de Política Monetária. Vários números. Disponível em: < http://www.bacen.gov.br >. Acesso em: 10 de março de 2012.

BARBOSA, F. H; SOARES, J.J.S. Regra de Taylor no Brasil: 1999-2005. In: ENCONTRO DA ANPEC, 2006.

BARCELLOS NETO, P. C. F. de.; Portugal, M. S. Determinants of monetary policy committee decisions: Fraga vs. Meirelles. Porto Alegre: PPGE/UFRGS, 2007. (Texto para Discussão, 11).

BARCELLOS NETO, P. C. F. de. Estimando uma regra de Taylor para o sistema de metas de inflação brasileiro. I Prêmio Banco Central de Monografias em Política Monetária, 2003. Disponível em: <http:/www.bacen.gov.br>.

CHOW, G. C. Tests of Equality between Sets of Coefficients in Two Linear Regressions. Econometrica, v.28, p.591-605, 1960.

CLARIDA, R.; GALÍ, J.; GERTLER, M. Monetary policy rules and macroeconomic stability: evidence and some theory, forthcoming. Quarterly Journal of Economics, 2000.

CLARIDA, R.; GALÍ, J.; GERTLER, M. Monetary policy rules in practice: some international evidence. European Economic Review, v. 42, p. 1033-1067, 1998.

GREENE, W. H. Econometric analysis. 5.ed. New Jersey: Prentice Hall, 2003.

GUJARATI, D. N. Econometria Básica. 4. ed. Rio de Janeiro: Elsevier, 2006.

HOLLAND, M. Monetary and exchange rate policy in Brazil after inflation targeting. Berkeley: University of California, 2005.

INSTITUTO DE PESQUISA ECONÔMICA APLICADA. Disponível em: <http:// www.ipeadata.gov.br>. Acesso em: og de março de 2012.

JUDD, J.; RUDEBUSCH, G. Taylor's rule and the FED: 1970-1997. Federal Reserve Bank of San Francisco. Economic Review, v. 3, p. 3-16, 1998.

KWIATKOWSKI, D.; PHILLIPS, P. C. B.; SCHMIDT, P; SHIN, Y. Testing the null hypothesis of stationary against the alternative of a unit root. Journal of Econometrics, v. 54, 1992.

LIMA, E. C. R.; MAKA, A.; MENDONÇA, M. Monetary policy regimes in Brazil. Instituto de Pesquisa Econômica Aplicada (IPEA). Rio de Janeiro, n. 1285a, jun. 2007. (Texto para Discussão). Disponível em: <http:/www.ipea.gov.br> .

MEDEIROS, G. B.; ARAGÓN, E. K. S. B. Testando Assimetrias nas Preferências do Banco Central em uma Pequena Economia Aberta: Um Estudo para o Brasil. In: XVI Encontro de Economia Regional. Anais. Fortaleza, 2011. 
MINELLA, A. et al. Inflation targeting in Brazil: construction credibility under exchange rate volatility. Brasília: Banco Central do Brasil, 2003. (Trabalhos para Discussão, 77).

OLIVEIRA, N. S. M. N.; ARAGÓN, E. K. S. B. Testando Quebra Estrutural na Regra de Taylor: Um Estudo Empírico para o Brasil (200O-2009). In: V Encontro de Economia Catarinense. Anais. Florianópolis, 2011.

POLICANO, R. M.; BUENO, R. D. L. S. A sensibilidade da política monetária no Brasil: 1999-2005. In: BRAZILIAN ANNUAL MEETING OF ECONOMY, 34., 2006. Salvador.

SALGADO, M. J. S. et al. Monetary policy during Brazil's Real Plan: estimating the central bank's reaction function. Revista Brasileira de Economia, v. 59, n. 1, 2005 .

SURICO, P. The monetary policy of the European Central Bank. Scand. Journal of Economics, v. 109, n. 1, 2007.

TAYLOR, J. Discretion versus policy rules in practice. Carnegie-Rochester Conference on Public Policy, v. 39, p. 195-214, 1993.

Recebido em: 05 de abril de 2013

Aceito em : 07 de janeiro de 2014 